\title{
Definición de un método para la programación de la producción desde el paradigma de los sistemas holónicos de manufactura
}

INDUSTRIAL ENGINEERING, MANUFACTURING SYSTEMS

\section{Defining a method for production scheduling from holonic manufacturing systems paradigm}

\author{
Diego A. Burbano ${ }^{*}$, John D. López ${ }^{*}$, Oscar A. Rojas \\ 'Departamento de Electrónica, Instrumentación y Control, Universidad del Cauca. \\ Popayán, Cauca, Colombia \\ ${ }^{* *}$ Grupo de I+D en Automática Industrial, Departamento de Electrónica, Instrumentación y Control, \\ Universidad del Cauca. Popayán, Cauca, Colombia \\ diegobq@unicauca.edu.co,sjohndlopez@unicauca.edu.co,orojas@unicauca.edu.co
}

(Recibido: Diciembre 18 de 2014 - Aceptado: Julio 23 de 2015)

\begin{abstract}
Resumen
La globalización del mercado representa una ampliación en la visión de negocio de toda industria, el aumento del entorno empresarial constituye un reto en cuestión de competitividad pero a la vez una oportunidad de ingresar a nuevos mercados. Los mercados actuales definen los requerimientos de la industria, estos requerimientos reflejan un alto sentido de personalización, puesto que en la última década, el rápido cambio de los productos se está convirtiendo en un estilo de vida. Los clientes esperan una mejor calidad, mayor variedad y mejor rendimiento de los productos (Palominos, 2007). Es así como los sistemas de manufactura buscan un nuevo enfoque que se adecue eficientemente a estas necesidades. Puntualmente, la función de programación de la producción representa un desafío estrechamente ligado a las tendencias del sector, la asignación de recursos debe desarrollarse de forma flexible, de tal manera que se adecue a las necesidades de producción dinámicas. Considerando lo anterior, en el presente artículo se presenta un método para la programación de la producción desde el paradigma de los sistema holónicos de manufactura enfatizando en la definición de tareas, interfaces y flujos de información, así como también la aplicación de modelos para la estructuración del método.
\end{abstract}

Palabras clave: Estándares en automatización, modelado estructural, programación de la producción, sistemas holónicos de manufactura.

\begin{abstract}
Marketing globalization represents an expansion of the business vision of the whole industry, increased business environment is a challenge within competitiveness but also an opportunity to enter new markets. Current markets define the industry requirements, these requirements reflect a high sense of customization, since in the last decade, the rapid change of products is becoming a lifestyle. Customers expect better quality, greater variety and better product performance. Thus, as manufacturing systems are in search of a new approach that efficiently adapts to these needs. Specifically, the function of production scheduling in the manufacturing system represents a challenge closely linked to industry trends, the allocation of resources should be developed in a flexible way, such that suits the dynamics production needs. Considering the above, in this paper a method for production scheduling from the paradigm of holonic manufacturing system is proposed, emphasizing the definition of tasks, interfaces and
\end{abstract}


information flows as well as the application of models for structuring the method.

Keywords: Automation standard, holonic manufacturing system, programming production, structural modeling.

\section{Introducción}

En años recientes, muchas tendencias han sido establecidas en la relación sociedad-manufactura, concretamente: globalización del mercado, creciente personalización del producto o servicio, aumento en la complejidad tecnológica, aumento del número de competidores, disminución del ciclo de vida del producto, y aumento en requerimientos de calidad (Sousa \& Ramos, 2004). Para poder adaptarse a estas tendencias, la industria manufacturera necesita renovar su visión, configurar su estructura organizacional de tal manera, que se alcance un sistema de producción con alto grado de flexibilidad operacional. En respuesta a estas exigencias, han surgido nuevos paradigmas de producción que se desarrollan dentro del enfoque de la nueva generación de manufactura, como lo son el paradigma heterárquico y holárquico. No obstante muchos de los esquemas de producción actuales aún se fundamentan en el paradigma jerárquico, el cual presenta una estructura de múltiples niveles, coordinados por una entidad de orden superior. En el contexto actual, los sistemas tradicionales no cuentan con una estructura que les permita adaptarse a las tendencias del sector manufacturero debido a su naturaleza rígida. El paradigma heterárquico posee atributos que le permiten desempeñarse en un entorno de mercado inestable, pero debido a la falta de un ente coordinador, la eficiencia de los objetivos globales de producción se ve afectada considerablemente. Por eso, se hace necesario el desarrollo de una visión que combine los fundamentos tradicionales del paradigma jerárquico con el enfoque innovador del paradigma heterárquico. Es así como surge la noción de sistemas holónicos de manufactura (HMS), compuestos por entidades llamadas holones, que se organizan en jerarquías temporales reconfigurables llamadas holarquías, mediante las cuales se provee flexibilidad y adaptabilidad, reaccionando ágilmente a perturbaciones, preservando la estabilidad y optimización global del sistema de manufactura (Zapata, 2011).

Por otro lado, una de las funciones principales de un sistema de manufactura, es la programación de la producción, la cual representa un elemento clave en la eficiencia productiva global. La programación de la producción se define como una función inmersa en el nivel de administración de operaciones de manufactura (ISA 95.00.03, 2005), junto con los ámbitos de calidad, inventario y mantenimiento. Según (Bongaerts, 1998), la programación de la producción se define como el proceso de optimización de las decisiones de asignación de recursos.

Así, en el presente trabajo se presenta un método para la programación de la producción desde el paradigma de los sistemas holónicos, enfocándose en el cumplimiento de los requerimientos de las tendencias identificadas como claves en el sector manufacturero. Como primera medida se realiza un análisis funcional con base en estándares en automatización, el cual tiene como objetivo primordial puntualizar las tareas, interfaces y flujos de información necesarios para soportar el método, a partir de estándares, posteriormente se presentan las principales características de los sistemas holónicos de manufactura, para finalmente establecer el método, en base a. los conceptos concretados en el análisis funcional, abordados desde las nociones holónicas, enfatizando en el modelado estructural mediante la técnica IDEF0, y el modelado dinámico a través de la técnica WF-Net.

\section{Marco teórico}

\subsection{Conceptualización de la programación de la producción}

En la literatura en general, se observa una divergencia de enfoques respecto a la función de programación de la producción, lo cual se ve reflejado en la heterogeneidad de tareas, modelos, métodos, procedimientos, etc. desarrollados para 
esta función en cada empresa. Por esta razón se hace necesario la aplicación de estándares en automatización para conceptualizar dicha función, en el presente trabajo se toma como referencia los conceptos definidos en el estándar ANSI/ISA-95.

Como primera medida se referencia el Modelo jerárquico funcional expuesto en la parte 1 del estándar ISA-95 (2000), la Figura 1 muestra los diferentes niveles definidos en este modelo: logística y planificación de negocios; administración de operaciones de manufactura, y el nivel de control batch, continuo o discreto. En el nivel 4 se define un programa de producción básico o primario, el cual se elabora en base a la capacidad de producción, niveles de inventario y materias primas, así como también plazos de entrega, costos y otros aspectos propios de este nivel. La función de programación de la producción se encuentra dentro de las actividades de nivel 3, y recibe del nivel 4 el programa básico de producción, y a partir de este genera un programa detallado de producción, enmarcado en un horizonte de tiempo a corto plazo, el cual tiene en cuenta la información actual de la planta de producción, con el objetivo de realizar una asignación óptima de recursos. Posteriormente se envía a los niveles inferiores de control y monitoreo, el documento guía para la ejecución del programa detallado de producción.

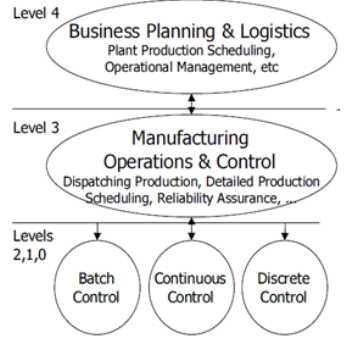

Figura. 1. Modelo jerárquico funcional.

A continuación se enfatiza en las actividades de nivel 3, para ello se hace uso del Modelo de flujo de datos funcional definido en la parte 3 de la norma ISA-95 (2005), este modelo describe la interfaz presente entre los niveles 3 y 4, profundizando en la definición de los flujos de información intercambiados en esta frontera, así como también flujos internos dentro del nivel 3.

Por último se referencia el Modelo de actividades de administración de operaciones de producción con el objetivo de identificar las principales tareas definidas por el estándar ISA-95 para la función de programación de la producción, y profundizar en los flujos de información internos intercambiados en las actividades del ámbito de producción.

\subsection{Análisis funcional del método para la programación de la producción}

En base a los conceptos expuestos anteriormente, se realiza un análisis funcional del método para la programación de la producción, el cual tiene como objetivo concretar interfaces, tareas y flujos de información. En la Figura 2 se establecen las interfaces internas y externas de la función de programación de la producción con los niveles 2 y 4 del modelo jerárquico funcional, resaltando el flujo de información principal que se intercambia internamente entre las sub-funciones de programación, programación detallada $\mathrm{y}$ despacho de la producción. Del nivel 4, se recibe una orden de producción, la cual contiene como información primordial el tipo de producto a elaborar, la cantidad y el plazo de entrega. La sub-función programación de la producción recibe dicha información, y en base a la regla de producción del tipo de producto de la orden está en la capacidad de generar un programa de producción, el cual contiene la secuencia de operaciones necesarias para la elaboración de dicho producto, definiendo tiempos de procesamiento, materias primas requeridas y otras especificaciones, en esta etapa aún no se asigna un recurso en específico para cada operación. La sub-función programación detallada de la producción recibe el programa de producción y en base a los requerimientos operacionales aquí definidos, desarrolla el proceso de asignación de recursos, generando un programa detallado de producción, el cual contiene la distribución optima de operaciones en el tiempo junto con su respectiva asignación de recursos, además de otra información definida anteriormente. 


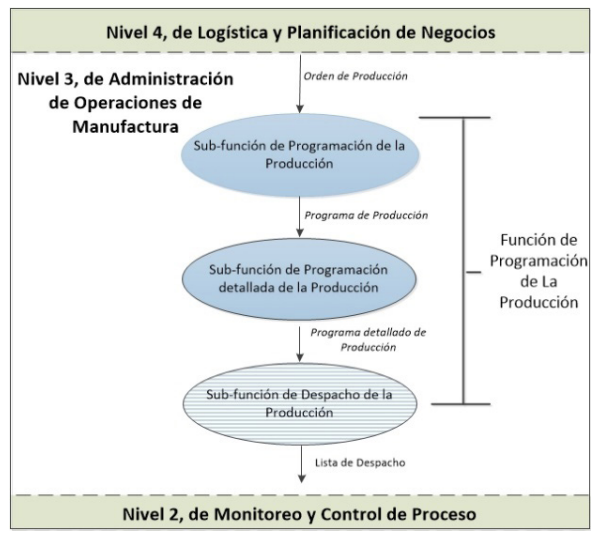

Figura. 2. Interfaz del método de programación de la producción

Las tareas que aplican para el método de programación de la producción desarrollado en el presente trabajo son:

- Creación y gestión del programa detallado de producción: El objetivo principal del método es establecer programas factibles, en base a la capacidad y disponibilidad de los recursos, reglas de producción, y otros criterios como costos de producción y plazos de entrega.

- Estado y asignación de recursos: Administra la información sobre el estado de los recursos, incluyendo la capacidad y disponibilidad de los mismos, asegurando que la función de asignación de recursos se desarrolle eficientemente.

- Monitoreo del programa detallado de producción: El método permite realizar un seguimiento continuo del programa detallado de producción, verificando el estado actual y el cumplimiento de las fases u operaciones preestablecidas.

- Manejo de eventos no anticipados y reprogramación: En caso de ocurrir eventos que afecten el normal desarrollo del programa detallado de producción (fallas en equipos, inconvenientes con materias primas, ingreso y cancelación de órdenes de producción, etc.), el método está en la facultad de identificar estos incidentes y efectuar una función de reprogramación si es necesario.

- Establecer los flujos de información necesarios con los ámbitos de mantenimiento, calidad e inventario: Se requiere un flujo de información con el ámbito de mantenimiento con el fin de verificar la disponibilidad de equipos, basándose en el programa de mantenimiento. Con el ámbito de calidad se efectúa un intercambio de información con el fin de asegurar la calidad en productos, materias primas y el desarrollo de los procesos de producción. Con inventario se intercambia información referente al flujo de productos terminados.

- Generación de reportes de producción: Posterior a la ejecución del programa detallado de producción, el método está en la capacidad de recolectar y transmitir información sobre el desempeño de la producción para el respectivo análisis en un nivel superior.

Por ultimo en la Tabla 1 se especifican las interfaces y flujos de información que el método de programación de la producción precisa para su desarrollo.

\subsection{Sistemas Holónicos de Manufactura}

En esta sección se presentan las principales características de los sistemas holónicos de manufactura (HMS), los cuales combinan la estabilidad del enfoque jerárquico con la flexibilidad dinámica del enfoque heterárquico, por lo tanto, son una alternativa prometedora frente a las tendencias de la industria. El término "holónico" es derivado de la palabra "holón", los cuales tienen cierto grado de independencia y manejan imprevistos sin consultar a autoridades superiores. Simultáneamente, los holones están sujetos a un control de autoridades superiores (Bongaerts, 1998). Dentro de los HMS se conforman jerarquías temporales para lograr metas específicas, en estas se definen reglas de cooperación entre los holones que restringen sus decisiones autónomas para asegurar desempeños controlables y predecibles en búsqueda de la optimización de recursos y respuesta en el tiempo que exigen las empresas de manufactura (Rojas \& Velasco, 2013). Una holarquía es definida como un conjunto de holones organizados en una estructura jerárquica, los cuales cooperan para alcanzar los objetivos del sistema, al combinar 
sus conocimientos y habilidades, como se ilustra en la Figura 3. Un holón puede pertenecer a múltiples holarquías al mismo tiempo, lo cual representa una diferencia significativa frente a los conceptos tradicionales de jerarquía. Los holones pueden integrarse a una holarquía, y al mismo tiempo, preservar su autonomía y singularidad (Leitão, 2004). Según el HMS Consortium los principales atributos de los sistemas holónicos de manufactura son:

- Autonomía: La capacidad de una entidad para crear y controlar la ejecución de sus propios planes.

- Cooperación: Proceso por el cual los holones desarrollan planes mutuamente aceptados, tras un proceso de acuerdo de misiones.

- Proactividad: Capacidad de anticiparse a cambios en sus planes y objetivos.

- Reactividad: Capacidad de reaccionar a estímulos del entorno.

Para abordar la función de programación desde el enfoque de los sistemas holónicos de manufactura se eligió la Unidad de Producción Holónica (UPH) como arquitectura de referencia, la cual define las directrices para el funcionamiento del sistema. La unidad de producción holónica es un modelos de referencia de producción de sistemas continuos presentado en (Chacón et al. 2002). El concepto de UPH se fundamenta en el modelo de Unidad de Producción (UP), en este modelo, Tabla 1. Flujos de información del método de programación de la producción

\begin{tabular}{|c|c|c|}
\hline \multicolumn{2}{|c|}{ INTERFAZ } & FLUJO DE INFORMACION \\
\hline \multirow{13}{*}{$\begin{array}{l}\text { Función de Pro- } \\
\text { gramación de la } \\
\text { Producción }\end{array}$} & \multirow{4}{*}{$\begin{array}{l}\text { Nivel } 4 \text {, de } \\
\text { Logística y } \\
\text { Planificación de } \\
\text { negocios }\end{array}$} & $\begin{array}{l}\text { Orden de producción: Contiene información de pedidos de clientes previamente confirmados, } \\
\text { define el tipo de producto a elaborar, la cantidad, el plazo de entrega y otras especificaciones } \\
\text { necesarias. }\end{array}$ \\
\hline & & $\begin{array}{l}\text { Capacidad de la producción: Contiene información referente a la capacidad de producción de } \\
\text { la planta (inalcanzable, disponible y comprometida) para hacer frente a las diversas demandas. }\end{array}$ \\
\hline & & $\begin{array}{l}\text { Regla de producción: Comprende la definición de una receta maestra, la cual contiene el pro- } \\
\text { cedimiento estándar para la elaboración de un tipo de producto en particular, detallado en una } \\
\text { secuencia de operaciones con tiempos y demás especificaciones necesarias. }\end{array}$ \\
\hline & & $\begin{array}{l}\text { Objetivos de costos de producción: Contiene información referente a objetivos de costos de } \\
\text { producción en términos de uso de recursos. }\end{array}$ \\
\hline & $\begin{array}{l}\text { Nivel 2, de } \\
\text { Monitoreo y } \\
\text { Control del } \\
\text { proceso }\end{array}$ & $\begin{array}{l}\text { Estado de los recursos: Contiene información referente a la disponibilidad de equipos (dis- } \\
\text { ponible, en uso, en fallo o reservado) y la capacidad de producción (por ejemplo, el volumen } \\
\text { de trabajo de una prensa por hora). Así como también la verificación de disponibilidad de } \\
\text { materias primas. }\end{array}$ \\
\hline & $\begin{array}{l}\text { Nivel 3, Ámbito } \\
\text { de Calidad }\end{array}$ & $\begin{array}{l}\text { Especificaciones de calidad: Contiene la información necesaria para el aseguramiento de la } \\
\text { calidad en materias primas, procesos y productos. }\end{array}$ \\
\hline & \multirow{2}{*}{$\begin{array}{l}\text { Nivel 3, Ámbito } \\
\text { de Manteni- } \\
\text { miento }\end{array}$} & $\begin{array}{l}\text { Programa de mantenimiento: Contiene la información referente a las rutinas de manteni- } \\
\text { miento programadas, con el fin de administrar eficientemente la asignación de recursos. }\end{array}$ \\
\hline & & Solicitud de Mantenimiento: Solicitud para realizar una función de mantenimiento. \\
\hline & Flujo Interno & $\begin{array}{l}\text { Programa de producción: Contiene la secuencia de operaciones necesarias para elaborar un } \\
\text { producto en específico, sin la asignación de recursos respectiva. }\end{array}$ \\
\hline & $\begin{array}{l}\text { Nivel 2, de } \\
\text { Monitoreo y } \\
\text { Control del } \\
\text { proceso }\end{array}$ & $\begin{array}{l}\text { Programa detallado de producción: Contiene la secuencia de operaciones necesarias para } \\
\text { elaborar un producto en específico, junto con la asignación de recursos respectiva. Establecien- } \\
\text { do prioridades, tiempos de ejecución, reserva de equipos y requerimientos de materias primas } \\
\text { a corto plazo, y otros aspectos relevantes de productividad. }\end{array}$ \\
\hline & \multirow{2}{*}{$\begin{array}{l}\text { Nivel 3, Ámbito } \\
\text { de Inventario }\end{array}$} & $\begin{array}{l}\text { Inventario de productos terminados: Contiene la recopilación de productos terminados, } \\
\text { disponibles para su comercialización. }\end{array}$ \\
\hline & & $\begin{array}{c}\text { Requerimientos de materia prima y energía: Contiene requerimientos de materia prima y } \\
\text { energía, necesarios en la ejecución de un programa de producción. }\end{array}$ \\
\hline & $\begin{array}{c}\text { Nivel 4, de } \\
\text { Logística y } \\
\text { Planificación de } \\
\text { negocios }\end{array}$ & $\begin{array}{l}\text { Desempeño de la producción: Contiene la información referente a los resultados de produc- } \\
\text { ción, especificando los datos reales obtenidos en la ejecución del programa de producción. }\end{array}$ \\
\hline
\end{tabular}


la UP se concibe como un conjunto de unidades elementales o recursos que son organizados y configurados de tal manera que permitan realizar los procesos de transformación en la cadena de valor, con el objetivo de obtener los productos exigidos. De esta forma, un proceso productivo puede ser visto como la agregación de unidades de producción cooperantes. La UPH toma sus propias decisiones respecto al cumplimiento de su objetivo, pero está obligada a informar su estado en relación al cumplimiento de una meta, o si ésta no se puede cumplir debido a una falla o errores en su comportamiento. Los componentes básicos de la unidad de producción holónica son (Zapata, 2011):

- Holón Recurso (HR): representa un recurso de producción, asociado a un equipo en específico. Está provisto de un sistema de toma de decisiones que le permite cooperar con otros y negociar sus metas a partir de su estado, competencias, capacidad y disponibilidad. La agregación de holones recurso forma la unidad de producción, que dentro de la concepción holónica está definida como una holarquía.

- Misión: Representa la secuencia de operaciones programadas, asociadas a la elaboración de un producto durante un periodo de tiempo, las cuales han sido negociadas y aceptadas por la unidad. Se encarga de la negociación de metas y su seguimiento.

- Ingeniería: Representa el conocimiento del holón para desarrollar los productos. Contiene los métodos de producción que indican cómo se obtiene un producto y los recursos requeridos.

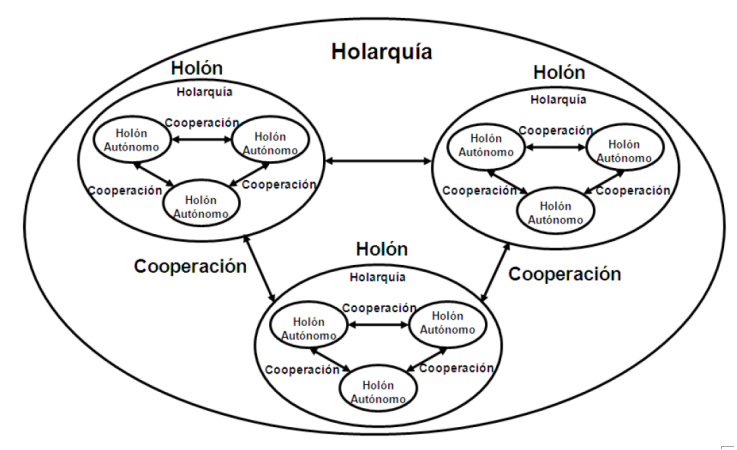

Figura. 3. Noción Holón-Holarquía.

\section{Resultados}

\subsection{Método para la programación de la producción desde los HMS}

En esta sección se busca unificar los conceptos holónicos con las especificaciones del método para la programación de la producción concluidas en el análisis funcional. Al mismo tiempo que se desarrolla la programación de la producción bajo la arquitectura UPH, se realiza el modelado estructural, el cual permite visualizar las relaciones e interacción entre los componentes de dicho sistema (Burbano \& López, 2014). Con el fin de profundizar en el intercambio de información y la interacción entre las actividades desarrolladas por el método, se aplica la técnica de IDEF0, que permite visualizar con mayor detalle la estructura del método de programación de la producción holónico. En la Figura 4 se modelan las sub-actividades que componen la función global de programación de la producción. En este modelo se especifican los flujos internos de información necesarios para el desarrollo de dicha función, en un entorno de fabricación holónico (UPH), se observa, además de las actividades involucradas en la programación de la producción, la interfaz con el nivel 4, mediante la actividad Procesamiento de Ordenes, y la interfaz con nivel 2 a través de la actividad de Despacho de la Producción. Las 4 actividades principales asociadas al método para la programación de la producción desde el enfoque HMS son: $S u b$ función de Programación de la Producción; Proceso de Asignación (acuerdo); Configuración de la Producción; Monitoreo y Reconfiguración del Programa detallado de Producción. 


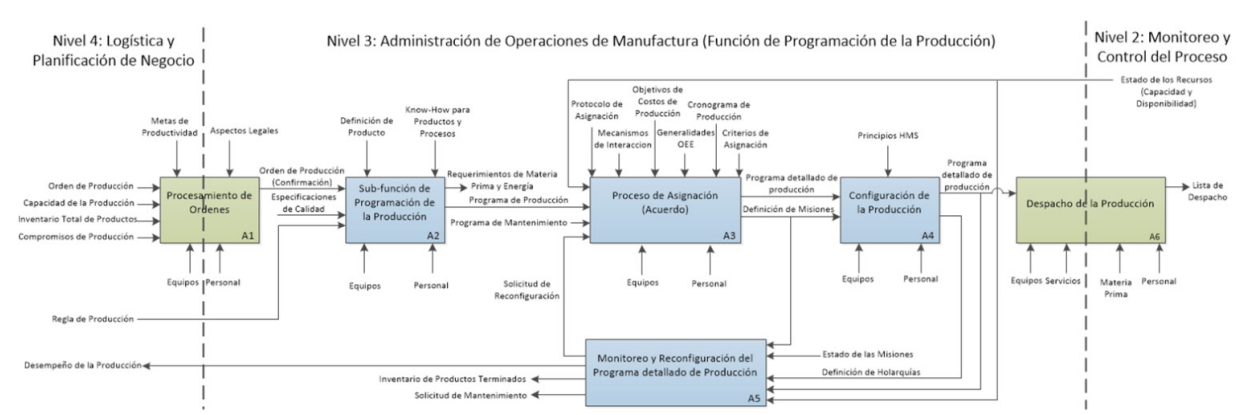

Figura. 4. Diagrama IDEFO (A0) para la función de programación de la producción desde el enfoque holónico.

\subsubsection{Sub-función de programación de la producción}

La sub-función de programación de la producción (A2) es la primera actividad que se identifica en el modelo IDEF0 del método de programación de la producción de la Figura 4, el objetivo principal de esta actividad es la elaboración del programa de producción para la UPH a partir de la recepción de una orden de producción entregada por el nivel 4. Para mostrar la interacción entre los componentes de la UPH mientras se desarrolla la sub-función de programación de la producción, se aplica la técnica de modelado de UML, diagrama de secuencia. En la Figura 5 se expone dicho modelo.

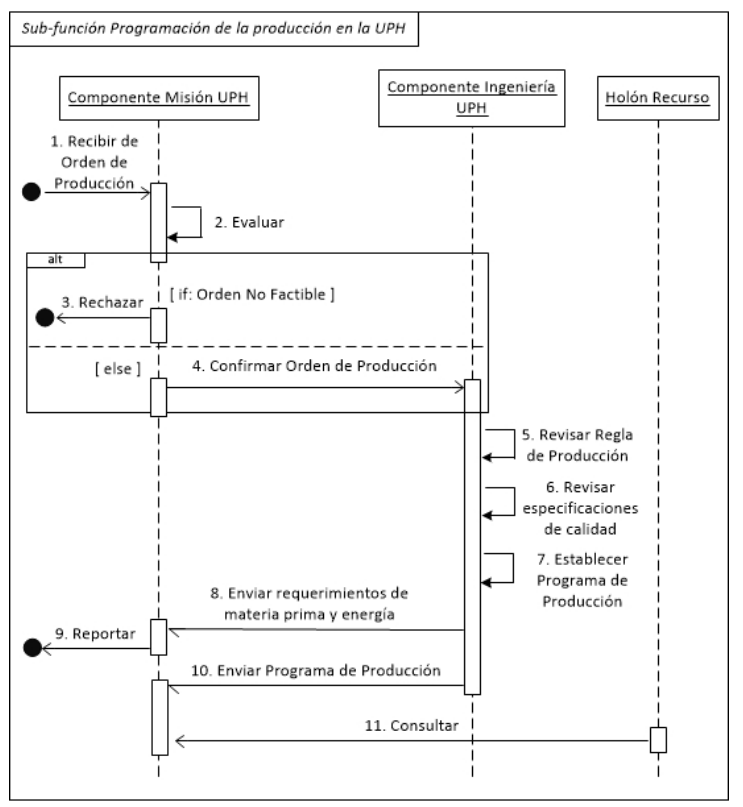

Figura. 5. Diagrama de secuencia sub-función programación de la producción.

\subsubsection{Proceso de Asignación (Acuerdo)}

El proceso de asignación (A3) es la segunda actividad dentro del modelo IDEF0, su objetivo principal es asignar recursos al programa de producción, dentro de la UPH este proceso consiste en la definición de una misión local para cada HR y su respectiva holarquía tras un proceso de acuerdo de operaciones.

El flujo de trabajo de la actividad A3 inicia con la recepción del programa de producción proveniente de la sub-función programación de la producción, este programa contiene los requerimientos operacionales necesarios para la fabricación de un producto específico, a partir de esta información el componente misión de la UPH inicia un proceso de solicitud a los holones para cumplir con estos requerimientos. Con el objetivo de desarrollar este proceso de forma eficiente, la UPH sigue un protocolo de asignación, el cual define las fases, el orden y los actores involucrados en dicho proceso. ContractNet es uno de los protocolos más utilizados en el proceso de asignación de recursos en un sistema de manufactura distribuido, fue propuesto por Smith (1990) y ha sido empleado para negociación y formación de holarquías en sistemas holónicos en múltiples trabajos. En la Figura 6 se expone el diagrama de secuencia del protocolo ContractNet aplicado en el proceso de asignación de recursos en la programación de la producción desde el enfoque holónico.

\subsubsection{Configuración de la producción}

La configuración de la producción (A4) es la tercera actividad dentro del modelo IDEF0, su objetivo principal es la formación de holarquías y configuración de los recursos de la UPH. En la 
actividad previa, se establece el programa detallado de producción como elemento concluyente del protocolo de asignación, en dicho programa se definen y se distribuyen en el tiempo las misiones para cada uno de los holones recurso de la UPH. Una vez confirmada la misión para cada HR, se procede a desarrollar el proceso de formación de holarquías, como se definió anteriormente una holarquía es una agrupación de holones recurso, estructurados en una jerarquía temporal, orientados al cumplimiento de una misión, para el caso de la función de programación de la producción, la agrupación de HR en una holarquía se efectúa en base a las órdenes de producción. En la Figura 7 se observa el diagrama de secuencia que modela la interacción de los componentes de la UPH en el desarrollo de la actividad A4.

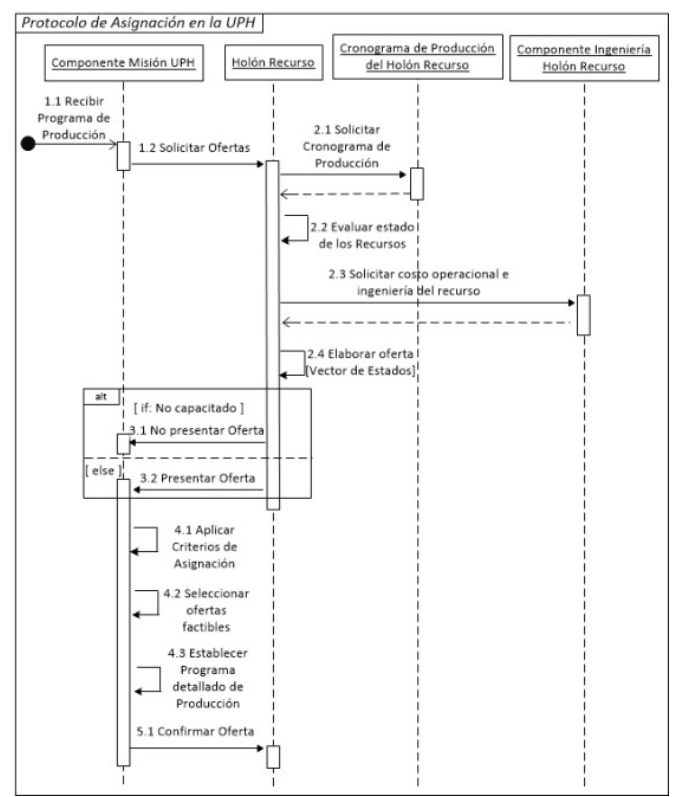

Figura. 6. Diagrama de secuencia Protocolo ContractNet.

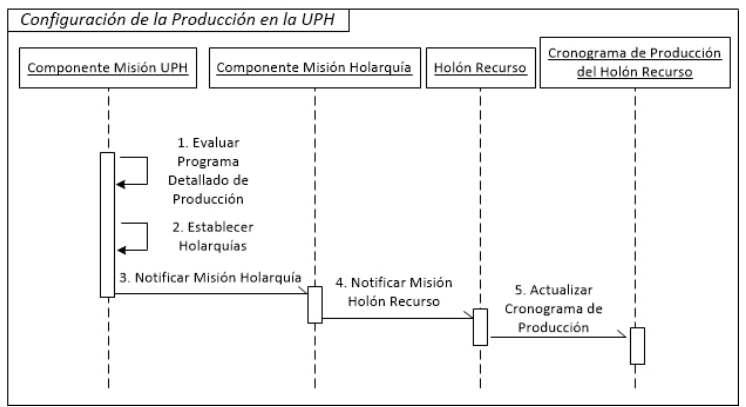

Figura. 7. Diagrama de secuencia configuración de la producción.

\subsubsection{Monitoreo y reconfiguración del programa detallado de producción}

La cuarta actividad en el modelo IDEF0 reúne los procesos de monitoreo y reconfiguración de la producción (A5), el principal objetivo de esta actividad es asegurar el cumplimiento de las metas de producción. Para ello se monitorea constantemente el avance de las misiones designadas para cada holón recurso, cada HR efectúa internamente este seguimiento y está en la responsabilidad de notificar al componente misión de su holarquía cualquier falla o evento no anticipado que ponga en riesgo el cumplimiento de su cronograma. En la Figura 8 se observa el diagrama de secuencia para el monitoreo de la producción en la UPH, haciendo énfasis en el manejo de fallas en el sistema, y en el procesamiento final que se le da a una orden de producción completa.



Figura. 8. Monitoreo y Reconfiguración de la producción en la UPH

3.2 Modelado dinámico del método para la programación de la producción

La presente sección tiene como objetivo visualizar la dinámica en la UPH, cuando se efectúa el flujo 
de trabajo definido previamente, para el método de programación de la producción. Para ello se utiliza la técnica de modelado Workflow-Net, la cual combina los conceptos de workflow con las bases de modelado de sistemas a eventos discretos de la redes de Petri. El modelado dinámico se desarrolla en base a los modelos estructurales obtenidos en la sección previa.

En la Figura 9 se observa el modelo WF-Net para la sub-función de programación de la producción (A2), allí se visualiza la interacción entre el componente misión de la UPH y su componente ingeniería. En la Figura 10 se expone el modelo
WF-Net de la configuración de la producción (A4), en la cual interactúan el componente misión de la UPH y el de la Holarquía, así como también el holón recurso. En base al programa detallado de producción definido en la actividad previa A3, el componente misión de la UPH establece holarquías junto con su respectiva misión. Por su parte el componente misión de cada holarquía establece las misiones locales para cada uno de los HR que la componen, y en base a ello el HR actualiza su cronograma de producción. En la Tabla 2 se indica la nomenclatura utilizada en las tareas de los modelos WF-Net.

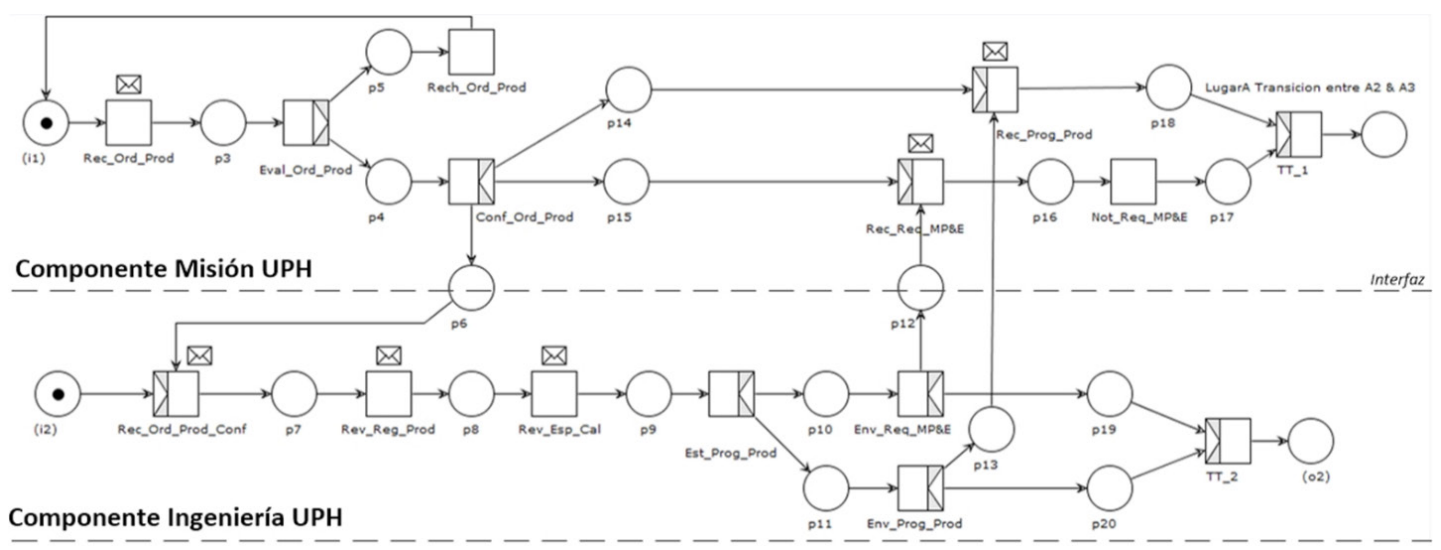

Figura. 9. WF-Net de la Sub-función programación de la producción (A2)

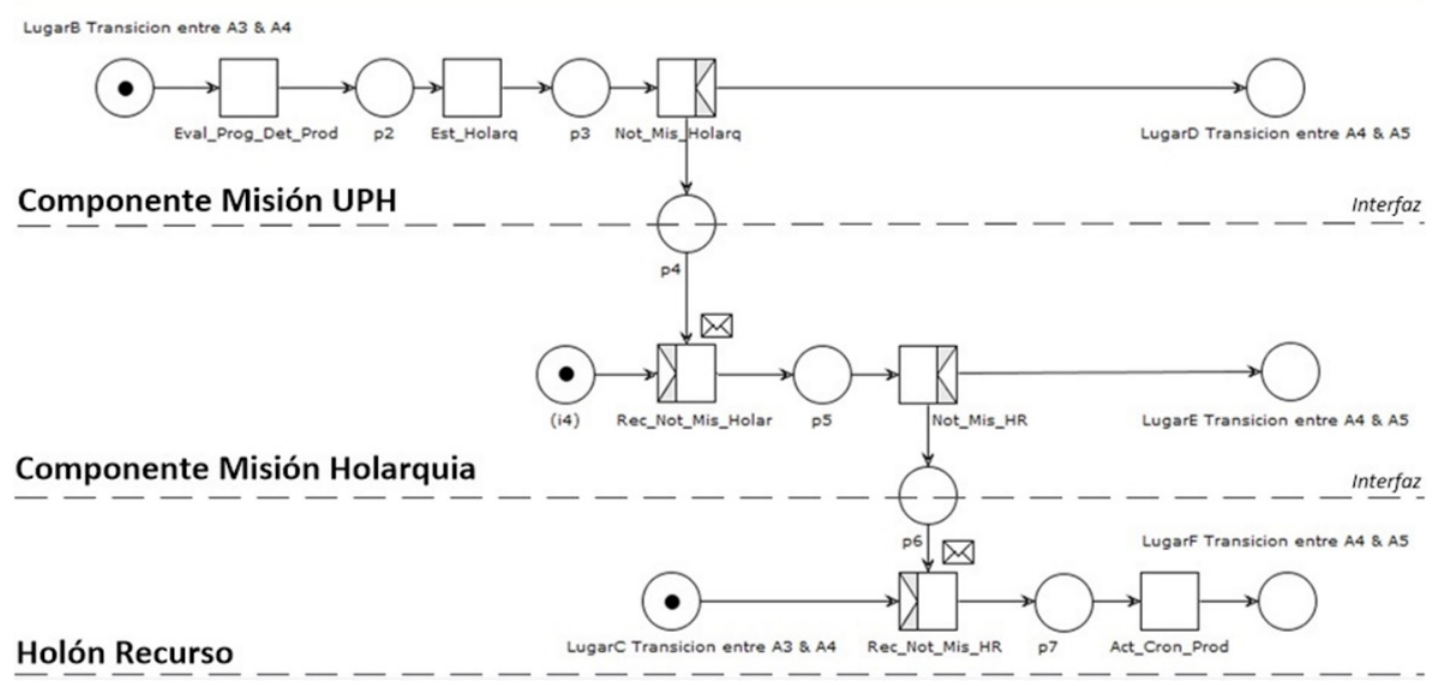

Figura. 10. WF-Net de la configuración de la producción (A4). 
Tabla 2. Nomenclatura empleada en los modelos WF-Net.

\begin{tabular}{|c|c|}
\hline NOMENCLATURA & SIGNIFICADO \\
\hline \multicolumn{2}{|c|}{ Sub-función programación de la producción } \\
\hline Rec_Ord_Prod & Recibir Orden de Producción \\
\hline Eval_Ord_Prod & Evaluar Orden de Producción \\
\hline Rech_Ord_Prod & Rechazar Orden de Producción \\
\hline Conf_Ord_Prod & Confirmar Orden de Producción \\
\hline Rec_Ord_Prod_Conf & Recibir Orden de Producción Confirmada \\
\hline Rev_Reg_Prod & Revisar Regla de Producción \\
\hline Rev_Esp_Cal & Revisar Especificaciones de Calidad \\
\hline Est_Prog_Prod & Establecer Programa de Producción \\
\hline Env_Req_MP\&E & Enviar Requerimientos de Materia Prima y Energía \\
\hline Env_Prog_Prod & Enviar Programa de Producción \\
\hline Rec_Req_MP\&E & Recibir Requerimientos de Materia Prima y Energía \\
\hline Not_Req_MP\&E & Notificar Requerimientos de Materia Prima y Energía \\
\hline Rec_Prog_Prod & Recibir Programa de Producción \\
\hline TT_1 & Tarea Transitoria 1 \\
\hline TT_2 & Tarea Transitoria 2 \\
\hline \multicolumn{2}{|c|}{ Configuración de la producción } \\
\hline Eval_Prog_Det_Prod & Evaluar Programa detallado de Producción \\
\hline Est_Holarq & Establecer Holarquías \\
\hline Not_Mis_Holarq & Notificar Misión Holarquía \\
\hline Rec_Not_Mis_Holarq & Recibir Notificación Misión Holarquía \\
\hline Not_Mis_HR & Notificar Misión Holón Recurso \\
\hline Rec_Not_Mis_HR & Recibir Notificación Misión Holón Recurso \\
\hline Act_Cron_Prod & Actualizar Cronograma de Producción \\
\hline
\end{tabular}

Conelfindecomprobarsucorrectofuncionamiento, se validan los modelos desarrollados en la sección previa. Para efectuar dicho proceso de validación se usa el software Woped versión 3.1.0, el cual no solo permite visualizar los modelos, sino también realizar un análisis semántico de los mismos, identificando propiedades claves que una red de Petri correctamente estructurada debe cumplir.

\subsection{Análisis de resultados}

Como primera medida se concluye con un correcto modelado dinámico, a partir del proceso de validación se verifican propiedades como la vivacidad además de verificar que las redes de Petri construidas sean acotadas y reiniciables, lo cual garantiza a su vez un desarrollo eficiente de las tareas ejecutadas por el método en términos de secuencia de operaciones.
Posteriormente se hace uso de un caso de estudio para comprobar las características del método en un proceso de producción, se toma como referencia la elaboración de embutidos, este proceso de ejemplificación tiene como objetivo exponer la facilidad del uso de los conceptos y modelos planteados, así como la eficiencia del método en un entorno de manufactura. En la selección del caso de estudio se tuvo en cuenta que fuera posible aplicar nociones holónicas como la formación de holarquías, en base a la flexibilidad, agilidad y variabilidad propia del proceso productivo. Al aplicar las nociones del método en el caso de estudio, se observa, una correcta adaptabilidad de los modelos construidos, al igual que un desarrollo eficiente del programa detallado de producción, a partir de una orden, regla y recursos de producción de la planta, además 
de otros principios operacionales. Además de resaltar características funcionales del método como la flexibilidad y agilidad, respondiendo adecuadamente a perturbaciones del entorno. Los resultados de la fase de ejemplificación se pueden observar en su totalidad en (Burbano \& López, 2014).

Finalmente se resalta las ventajas que ofrece el método en un sistema de manufactura, se destaca la facilidad y el nivel práctico en la aplicación $\mathrm{y}$ adecuación de los modelos en un entorno de fabricación. Observando la coherencia entre los modelos, flujos de información e interfaces, así como también la eficiencia del método para desarrollar la programación de la producción

\section{Conclusiones}

- Después de identificar las tendencias que conducen el sector industrial, se resalta el paradigma holónico como una solución prometedora frente a los nuevos requerimientos de los sistemas de manufactura, en base a propiedades de los HMS como la autonomía y cooperación se mantiene un equilibrio entre la distribución de conocimiento, toma de decisiones, y la optimización global del sistema.

- En el presente trabajo se logró formular un método que desarrolla la función de programación de la producción desde el enfoque holónico, como resultando final se obtuvo un método genérico del cual se resaltan atributos holónicos como la autonomía, fundamentada en el trabajo orientado al cumplimiento de objetivos locales para cada holón, el monitoreo continuo de estas misiones locales le brinda al sistema los atributos de reactividad y proactividad, la propiedad de cooperación se observa en la configuración final de la UPH, los atributos de recursividad, auto-similaridad y escalabilidad se encuentra intrínsecos en la noción de formación de holarquías, y por ultimo las propiedades de agilidad y flexibilidad se fundamentan en la estructura misma de la UPH y la capacidad de reconfiguración de los recursos asignados en el programa detallado de producción.
- El método para la programación de la producción propuesto en el presente trabajo se fundamenta en la aplicación de modelos genéricos, estos modelos fueron desarrollados en base a técnicas de modelado estructural y dinámico como IDEF0, UML y WF-Net. El esquema IDEF0 permite identificar todos los flujos de información y las actividades involucradas en la función de programación de la producción, el diagrama de secuencia de UML muestra la interacción entre los componentes de la UPH para desarrollar las actividades previamente identificadas. Por otra parte, WF-Net permite modelar y validar el flujo de trabajo que lleva a cabo el método para desarrollar la función de programación desde el enfoque holónico. Se resalta así, la importancia de la fase de modelado dentro de cualquier proyecto en el sector industrial.

\section{Referencias}

ANSI/ISA-95.00.01, (2000). Enterprise Control System Integration Part 1: Models and Terminology. Carolina Del Norte, USA, American National Standard.

ANSI/ISA-95.00.03, (2005). Enterprise Control System Integration Part 3: Activity Models of Manufacturing Operations Management. Carolina Del Norte, USA, American National Standard.

Bongaerts, L. (1998). Integration of Scheduling and Control in Holonic Manufaturing Systems. Tesis Doctoral, Katholieke Universiteit Leuven. Heverlee (Leuven), Bélgica.

Burbano, D., y López, J. (2014). Definición de un Método para la Programación de la Producción desde el Paradigma de los Sistemas Holónicos (HMS). Tesis de Pregrado, Universidad del Cauca, Popayán, Colombia.

Chacón, E., Besembel, I., Narciso, F., Montilva, J. \& Colina, E. (2002). An integration architecture for the automation of continous production complexes. ISA Transactions. Journal of the Americna Intitute of Physics 41(1), 95-113. 
Leitão, P. (2004). An Agile and Adaptive Holonic Architecture for Manufacturing Control. Tesis Doctoral, University of Porto. Porto, Portugal.

Palominos, P. (2007). Personalización en Masa: ¿Una Nueva Forma de Producción? Contribuciones Cientificas y Tecnológicas 43 (134), 20-30.

Rojas, O., Velasco, M., \& Chacon, E. (2013). Principios de un Modelo Dinámico para Integración Empresarial: Un enfoque desde los Sistemas Holónicos de Manufactura - HMS. Popayán, Colombia.

Smit, R. (1990). The Contract Net Protocol: HighLevel Communication and Control in Distribuited
Problem Solver. IEEE Transactions on Computers 29 (12), 104-1113.

Sousa, P., Ramos, C., \& Neves, J. (2004). The Fabricare system: a multi-agent-based scheduling prototype. Production Planning \& Control 15 (2), 156-165.

Zapata, G. (2011). Propuesta para la planificación, programación, supervisión y control de la producción en procesos continuos desde la teoría del control supervisorio y el enfoque holónico. Tesis Doctoral, Universidad de Los Andes. Mérida, Venezuela.

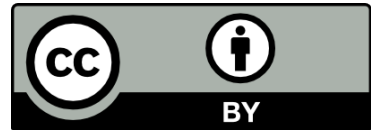

Revista Ingeniería y Competitividad por Universidad del Valle se encuentra bajo una licencia Creative Commons Reconocimiento - Debe reconocer adecuadamente la autoría, proporcionar un enlace a la licencia e indicar si se han realizado cambios. Puede hacerlo de cualquier manera razonable, pero no de una manera que sugiera que tiene el apoyo del licenciador o lo recibe por el uso que hace. 